\title{
Content-Based Image Retrieval By Relevance Feedback *
}

\author{
Zhong Jin ${ }^{1}$, Irwin King ${ }^{2}$, and Xuequn Li \\ 1 Department of Computer Science, \\ Nanjing University of Science and Technology, \\ Nanjing, People's Republic of China \\ jinzhong@mail.njust.edu.cn \\ 2 Department of Computer Science and Engineering, \\ The Chinese University of Hong Kong, \\ Shatin, N.T., Hong Kong \\ \{king, xqli\}@cse.cuhk.edu.hk
}

\begin{abstract}
Relevance feedback is a powerful technique for content-based image retrieval. Many parameter estimation approaches have been proposed for relevance feedback. However, most of them have only utilized information of the relevant retrieved images, and have given up, or have not made great use of information of the irrelevant retrieved images. This paper presents a novel approach to update the interweights of integrated probability function by using the information of both relevant and irrelevant retrieved images. Experimental results have shown the effectiveness and robustness of our proposed approach, especially in the situation of no relevant retrieved images.
\end{abstract}

\section{Introduction}

Content-based image retrieval(CBIR) has become one of the most active research areas in the past few years [1]-[6]. Generally, a set of features (e.g. color, shape, texture, moments, etc.) is extracted from an image to represent its content. Successful content-based image retrieval systems require the integration of various techniques in the fields of image processing and information retrieval [7].

Relevance feedback in information retrieval is an automatic process for query reformulation [8]-[10]. In principle, relevance feedback is based on automatically changing the set of query terms as well as the weights associated with these terms. This is done in order to increase the weights of important query terms and to decrease the weights of unimportant ones. It is accomplished by investigating the set of documents initially retrieved, and increasing the weights of those terms that are in the relevant documents. Experiments show the relevance feedback techniques considerably improve the quality of the term-weighting retrieval system.

* This paper is supported in part by an Earmarked RGC Grant from the Hong Kong Research Grant Council \# CUHK4176/97E. 
Recently, relevance feedback based CBIR techniques have emerged as a promising research direction. MARS [7] introduced both a query vector moving technique and a re-weighting technique to estimate the ideal query parameter. MindReader [11] formulated a minimization problem on the parameter estimation process. Rui et al. [12] present a relevance feedback based interactive retrieval approach, which effectively takes into account the following two distinct characteristic of CBIR systems: the gap between high-level concepts and low-level features, and subjectivity of human perception of visual content. Rui and Huang [13] proposed a novel global optimization framework. Wood, Campbell and Thomas [14] described an image database query system(IDQS), which consisted of a set of radial basic function (RBF) networks.

Most relevance feedback based CBIR techniques have only utilized information of the relevant retrieved images and cannot deal with situations of no relevant retrieved images. They have not made great use of information of the irrelevant retrieved images, especially in the situation of there is less relevant retrieved images than irrelevant retrieved images.

In our opinion, both the relevant retrieved images and the irrelevant retrieved images contain much information of features used. This paper presents a novel update approach for interweights to. It is organized as follows. In section 2, some relevance feedback techniques are introduced. A novel update approach for interweights is proposed in section 3 . In Section 4, performance experiments are conducted, and experimental results are discussed. Section 5 gives a conclusion.

\section{Overall Similarity Function}

Suppose an image databases $D B$ is composed of $c$ training images $\left\{I_{1}, I_{2}, \cdots, I_{c}\right\}$. For a query image $Q$, a retrieval decision can be made according to the overall similarity between $Q$ and any image $I \in D B$. An image contains rich content, such as color, shape, texture and invariant moments of the object in the image, and each of these features(e.g. shape) can be characterized by a feature vector.

Feature Extraction For any image $I \in D B$, a feature extraction function $F$ is defined as

$$
F(I): \Re^{|I|} \rightarrow \Re^{n}
$$

where $|\cdot|$ indicates the number of elements of a digital image matrix.

Suppose $m$ feature vectors can be extracted for an image, the similarity for the $i^{t h}$ feature between the query image $Q$ and any training image $I \in D B$ can be defined as followings:

$$
\Phi_{i}(I, Q)=\left(F_{i}(I)-F_{i}(Q)\right)^{T} W_{i}\left(F_{i}(I)-F_{i}(Q)\right)
$$

where $F_{i}(I)$ and $F_{i}(Q)$ are the $i^{t h} n_{i}$-dimensional image feature vectors of the images $I$ and $Q$, and $W_{i}$ are the $\left(n_{i} \times n_{i}\right)$ intraweight matrix associated with the components of the $i^{t h}$ feature vectors. Therefore, the overall similarity function 
between the query image $Q$ and any training image $I \in D B$ can be defined:

$$
D(I, Q)=\sum_{i=1}^{m} U_{i} \Phi_{i}(I, Q)
$$

where $U_{i}$ are the interweights associated with the $i^{\text {th }}$ feature vectors.

The images in the database $D B$ are ordered by their overall similarities to the query image $Q$. The $k$ most similar ones $\Re=\left\{R_{1}, R_{2}, \cdots, R_{k}\right\}$ are returned to the user, where $k$ is the number of images the user wants to retrieve.

Optimization Approach Suppose for any retrieved image $R_{j} \in \Re, \pi_{j}$ is the degree of relevance given by user. The following optimization problem was formulated by Y. Hui et al. [12]:

$$
\begin{aligned}
& \min J=\sum_{j=1}^{k} \pi_{j} D\left(R_{j}, Q\right) \\
& \text { s.t. }\left\{\begin{array}{l}
\sum_{i=1}^{m} \frac{1}{U_{i}}=1 \\
\operatorname{det}\left(W_{i}\right)=1 \\
(i=1, \cdots, m)
\end{array}\right.
\end{aligned}
$$

The optimal update for $Q_{i}$ is

$$
\hat{Q}_{i}=\frac{\sum_{j=1}^{k} \pi_{j} F_{i}\left(R_{j}\right)}{\sum_{j=1}^{k} \pi_{j}}
$$

That is, the ideal query vector for the $i^{t h}$ feature is nothing but the weighted average of the retrieved images for the $i^{\text {th }}$ feature.

The optimal solutions for $W_{i}$ is

$$
\hat{W}_{i}=\sqrt[n_{i}]{\operatorname{det}\left(C_{i}\right)} C_{i}^{-1}
$$

where $C_{i}=\sum_{j=1}^{k} \pi_{j} \Delta_{i j} \Delta_{i j}^{T} / \sum_{j=1}^{k} \pi_{j}, \Delta_{i j}=F_{i}\left(R_{j}\right)-F_{i}(Q)$. The physical meaning of this optimal solution is that the optimal weight matrix is inversely proportional to the covariance matrix of the retrieved images.

The optimal update formula for $U_{i}$ is

$$
\hat{U}_{i}=\sum_{v=1}^{m} \sqrt{J_{v} / J_{i}}
$$

where $J_{v}=\sum_{j=1}^{k} \pi_{j} \Delta_{v j}^{T} W_{v} \Delta_{v j}(v=1, \cdots, m)$. This formula tell us, if the total distance $J_{i}$ of the $i^{\text {th }}$ feature is small, this feature should receive high weight and vice versa.

For the interweight updating formula (8), $U_{i}$ are dealt only with the relevant retrieved images. This formula can not utilize any information of the irrelevant retrieved images, and does not work when all the retrieved images are regarded as irrelevant to the query by the user. This paper intends to make greater use of information of all the retrieved images. 
Scoring Approach Suppose for any retrieved image $R_{j} \in \Re$, the user marks it as highly relevant, relevant, neutral, irrelevant, or highly irrelevant, according to his information need and perception subjectivity. Let $\operatorname{Score}\left(R_{j}\right)$ be the relevance score fedback by the user for the $j^{t h}$ retrieved image $R_{j} \in \Re$ :

$$
\text { Score }\left(R_{j}\right)= \begin{cases}3 & \text { if highly relevant } \\ 1 & \text { if relevant } \\ 0 & \text { if no opinion } \\ -1 & \text { if irrelevant } \\ -3 & \text { if highly irrelevant }\end{cases}
$$

Let $\Re^{(i)}=\left\{R_{1}^{(i)}, R_{2}^{(i)}, \cdots, R_{k}^{(i)}\right\}$ be the set containing the $k$ most similar images to the query $Q$ according to the similarity value $\Phi_{i}(I, Q)$ for the $i^{\text {th }}$ feature. The interweights $U_{i}$ can be calculated with the following formula [13]:

$$
U_{i}=\sum_{R_{j} \in \Re^{(i)}} \operatorname{Score}\left(R_{j}\right)
$$

It is noted that if $U_{i}<0$, set it to 0 and the raw weights are needed to be normalized to make the sum of the normalized weights equal to 1.

\section{$3 \quad$ Integrated Probability Function}

Based on the posterior probability estimators, King and Jin [15] proposed an integrated probability function, which was successfully used as a new multifeature combination decision rule in the Chinese cursive script characteristic image retrieval.

Integrated Probability Function The following integrated probability function can serve as the overall similarity function $D(I, Q)[15]$ :

$$
D(I, Q)=\frac{\sum_{i=1}^{m} U_{i} P\left(F_{i}(I), F_{i}(Q)\right)}{\sum_{i=1}^{m} U_{i}}
$$

where $P\left(F_{i}(I), F_{i}(Q)\right)$ is the estimator of the posterior matching probability between image $I$ and image $Q$ on the $i^{\text {th }}$ feature, which is determined according to the following formula:

$$
P\left(F_{i}(I), F_{i}(Q)\right)=\frac{1}{c-1}\left(1-\frac{\left\|F_{i}(I)-F_{i}(Q)\right\|}{\sum_{v=1}^{c}\left\|F_{i}\left(I_{v}\right)-F_{i}(Q)\right\|}\right)
$$

where $\|\cdot\|$ indicates the common Euclidean distance.

Suppose for any retrieved image $R_{j} \in \Re$, the user marks it as relevant, neutral, or irrelevant according to his information need and perception subjectivity. Let $S$ be the number of retrieved images in $\Re$ which are relevant to the 
query $Q$ and $T$ be the number of retrieved images in $\Re$ which are irrelevant to the query $Q$.

For the $i^{\text {th }}$ feature, Let $s_{i}$ be the number of retrieved images in $\Re^{(i)}$ which are relevant to the query $Q$ and $t_{i}$ be the number of retrieved images in $\Re^{(i)}$ which are irrelevant to the query $Q$.

For the interweight updating formula (10), if the number of highly relevant or highly irrelevant retrieved images is supposed to be $0, U_{i}$ are dealt only with $s_{i}$ and $t_{i}$. When $S$ is much smaller than $T, s_{i}$ have more probabilities to be less than $t_{i}$, and therefore $U_{i}$ will be zero for each $i \in\{1,2, \cdots, m\}$. For example, suppose that $S$ be 1 and $T$ be 9 , then $s_{i}$ will be in $\{0,1\}$ and $t_{i}$ will be in $\{0,1, \cdots, 9\}$. However, it is easy to understand that in the above example, if for a given $i, s_{i}$ be 1 and $t_{i}$ be 2 , the $i^{t h}$ feature can be said to be more effective than all $m$ features to be used with known interweights, i.e., $U_{i}$ should be greater than 0, which is assigned according to the existing formula (10).

In our opinion, in order to make greater use of information of all the retrieved images, $U_{i}$ should be dealt with the ratios of $\frac{s_{i}}{S}$ and $\frac{t_{i}}{T}$. Taking into account the situations of zero relevant retrieved images or zero irrelevant retrieved images, a novel updating formula of raw interweights $U_{i}$ is presented as follows:

$$
U_{i}\left(s_{i}, t_{i}\right)=\exp \left\{\alpha\left(\frac{s_{i}+1}{S+1}-\frac{t_{i}+1}{T+1}\right)\right\}
$$

where $\alpha>0$ is a constant.

Obviously, $U_{i}\left(s_{i}, t_{i}\right)$ will increase as $s_{i}$ increases for a fixed $t_{i}$, and will decrease as $t_{i}$ increases for a fixed $s_{i}$. If for any fixed $i^{\text {th }}$ feature, $s_{i}=S$, and $t_{i}=T$, we have $U_{i}(S, T)=1$, which means that the $i^{t h}$ feature is as effective as all features. As we know, the exponential function $\exp (\cdot)$ changes slowly on the interval $(-1,1)$, a large parameter $\alpha$ is needed in the formula (13). The raw weights are needed to be normalized to make the sum of the normalized weights equal to 1 .

\section{Experimental Results}

In this section, we compare the retrieval performance between the proposed method and the scoring method.

There are 10 trademark images with $111 \times 111$ resolution [6]. For each trademark image, 10 deformed images and 10 hand draw images can be obtained, and these 21 images can be regarded as to be relevant images. The database is composed of 10 trademark images and their corresponding 10 deformed images for each. All the 110 images in the database $D B$ are shown in Figure 1. In the experiment, we tested our system with 100 hand drawn images, which are divided into 10 groups and indexed from 1 to 100 . Figure 2 shows these 100 hand drawn test images.

For an image, seven kinds of features are extracted. They are listed in Table 1. The first four kinds of features are from [6], and the last three kinds of features are computed on $55 \times 55$ low resolution images [15]. 


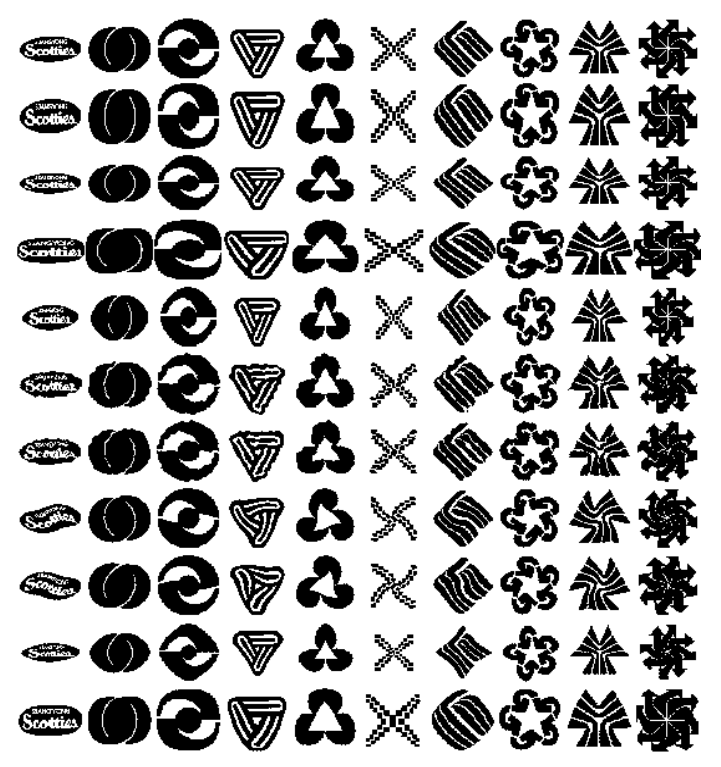

Fig. 1. 110 Images in the Database $D B$

The retrieval performance is measured using the following average retrieval precision:

$$
\text { Precision }=\frac{\text { relevant retrieved }}{k} \times 100 \%
$$

where $k=10$ is the number of total retrieved images.

The initial interweights $U_{i}(i=1, \cdots, 7)$ are given to be equal. The parameter $\alpha$ is chosen to be 5 . The average retrieval precisions for each test group and all the test images are summarized in Table 2. The symbol " $r f$ " denotes how many iterations of relevance feedback.

From Table 2, the average retrieval precision increases from $74.7 \%$ to over $90 \%$ by relevance feedback techniques. Relevance feedback is a powerful tool for content-based information retrieval. Moreover, the proposed update formula (13) is more effective than the existing scoring formula (10).

For 74 samples out of all 100 test images, the same retrieval performances are obtained with these two methods. For the other 26 samples, two methods obtain different retrieval performances. These experimental results in detail are listed in Table 3.

From Table 3, the proposed novel method is shown to be more robust than the scoring method, especially for $33^{\text {th }}$ sample. Moreover, it can make great use 


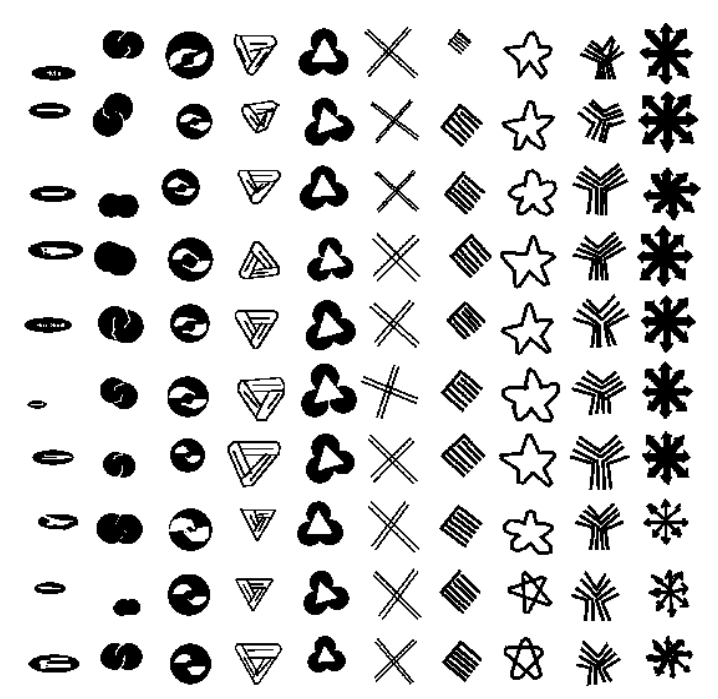

Fig. 2. 100 Hand Drawn Test Images

of information of both relevant retrieved images and irrelevant retrieved images, especially in the situations of no relevant retrieved images.

\section{Conclusion}

In this paper, a novel relevance feedback technique for updating the interweights has been proposed. Experimental results show that the proposed method outperforms the existing scoring method. Although our database is small, it is convincing that the proposed method can make great use of information of all the retrieved images to the user.

\section{Acknowledgement}

This paper is supported in part by a Earmarked RGC Grant from the Hong Kong Research Grant Council \#CUHK4407/99E.

\section{References}

1. C. Faloutsos, R. Barber, M. Flickner, J. Hafner, W. Niblack, D. Petkovic, and W. Equitz. Efficient and effective querying by image content. Journal of Intelligent Information Systems, 3(3/4):231-262, July 1994. 
Table 1. Seven Kinds of Features for an Image

\begin{tabular}{|c|l|}
\hline No. & \multicolumn{1}{|c|}{ Description of Feature } \\
\hline \hline 1 & 1-dimensional eccentricity $[6]$ \\
\hline 2 & 1-dimensional circularity of approximated boundary \\
\hline 3 & 7-dimensional invariant moment vector \\
\hline 4 & 63 -dimensional Fourier descriptors of approximated boundary \\
\hline 5 & 36 -dimensional pseudo Zernike moment vector $\left\{Z_{n k}(0 \leq k \leq n \leq 7)\right\}[15]$ \\
\hline 6 & 30 -dimensional Zernike moment vector $\left\{Z_{n k}(0 \leq k \leq n \leq 8\right.$, and $n-k=$ even $\left.)\right\}$ \\
\hline 7 & 25-dimensional Legendre moment vector $\left\{\lambda_{m n}(0 \leq m, n \leq 4)\right\}$ \\
\hline
\end{tabular}

Table 2. Average Retrieval Precisions in Percentage for Groups and for $D B$

\begin{tabular}{|c|c|c|c|c|c|c|c|c|c|c|c|}
\hline \multirow[b]{2}{*}{ Group } & \multirow[b]{2}{*}{$0 r f$} & \multicolumn{2}{|c|}{ Scoring } & \multicolumn{2}{|c|}{ Proposed } & \multirow[b]{2}{*}{ Group } & \multirow[b]{2}{*}{$0 r f$} & \multicolumn{2}{|c|}{ Scoring } & \multicolumn{2}{|c|}{ Proposed } \\
\hline & & $1 r f$ & $2 r f$ & $1 r f$ & $2 r f$ & & & $1 r f$ & $2 r f$ & $1 r f$ & $2 r f$ \\
\hline $1-10$ & 100 & 100 & 100 & 100 & 100 & $51-60$ & 100 & 100 & 100 & 100 & 100 \\
\hline $11-20$ & 81 & 100 & 100 & 100 & 100 & $61-70$ & 50 & 97 & 100 & 92 & 100 \\
\hline $21-30$ & 17 & 60 & 60 & 63 & 81 & $71-80$ & 63 & 91 & 92 & 95 & 95 \\
\hline $31-40$ & 98 & 94 & 99 & 99 & 99 & $81-90$ & 78 & 81 & 85 & 87 & 90 \\
\hline $41-50$ & 96 & 100 & 100 & 100 & 100 & $91-100$ & 64 & 77 & 77 & 83 & 85 \\
\hline \multicolumn{7}{|c|}{ Average Precision for $D B$} & 74.7 & $\overline{90}$ & 91.3 & 91.9 & 95 \\
\hline
\end{tabular}

2. C. P. Lam, J. K. Wu, and B. Mehtre. STAR-A System for trademark archival and retrieval. In 2nd Asian Conf. on Computer Vision, volume 3, pages 214-217, 1995.

3. Y. S. Kim and W. Y. Kim. Content-based trademark retrieval system using visually salient feature. In IEEE Computer Society Cnf. on Computer Vision and Pattern Recognition, pages 307-312, 1997.

4. A. K. Jain and A. Vailaya. Shape-based retrieval: A case study with trademark image databases. Pattern Recognition, 31(9):1369-1390, 1998.

5. J. P. Eakins, J. M. Boardman, and M. E. Graham. Similarity retrieval of trademark images. IEEE Multimedia, 5(2):53-63, 1998.

6. D. Y. M. Chan, I. King, D. P. Huijsmans et al. Genetic algorithm for weights assignment in dissimilarity function for trademark retrieval. In Visual Information and Information Systems. Third International Conference, VISUAL'99. Proceedings (Lecture Notes in Computer Science Vol.1614), pages 557-565, The Netherlands, June 1999.

7. Y. Rui, T. S. Huang, and S. Mehrotra. Content-based image retrieval with relevance feedback in MARS. Proceedings of IEEE International Conference on Image Processing,pp815-818, Santa Barbara, California, October, 1997.

8. I. J. Aalbersberg. Incremental relevance feedback. 15th International ACM/SIGIR Conference on Research and Development in Information Retrieval, Demark, June, 1992, pp11-22

9. C. Lundquist, D. A. Grossman, and O. Frieder. Improving relevance feedback in the vector space model. Proceedings of the Sixth International Conference on Information and Knowledge Management. CIKM'97, Las Vegas, Nevada, USA, Nov. 1997, pp16-23 
Table 3. Average Retrieval Precisions in Percentage for 26 Samples

\begin{tabular}{|c|c|c|c|c|c|c|c|c|c|c|c|}
\hline \multirow[b]{2}{*}{ Sample } & \multirow[b]{2}{*}{$0 r f$} & \multicolumn{2}{|c|}{ Scoring } & \multicolumn{2}{|c|}{ Proposed } & \multirow[b]{2}{*}{ Sample } & \multirow[b]{2}{*}{$0 r f$} & \multicolumn{2}{|c|}{ Scoring } & \multicolumn{2}{|c|}{ Proposed } \\
\hline & & $1 r f$ & $2 r f$ & $1 r f$ & $2 r f$ & & & $1 r f$ & $2 r f$ & $1 r f$ & $2 r f$ \\
\hline 23 & $\overline{0}$ & $\overline{\overline{0}}$ & $\overline{00}$ & $\overline{\overline{0}}$ & $\overline{10}$ & $\overline{77}$ & $\overline{660}$ & $\overline{70}$ & $\overline{770}$ & $\overline{880}$ & $\overline{880}$ \\
\hline 24 & 0 & 0 & 0 & 10 & 90 & 80 & 60 & 80 & 80 & 90 & 90 \\
\hline 26 & 0 & 0 & 0 & 20 & 100 & 82 & 80 & 90 & 90 & 70 & 80 \\
\hline 33 & 90 & 40 & 20 & 90 & 90 & 84 & 90 & 80 & 90 & 100 & 100 \\
\hline 61 & 60 & 100 & 100 & 90 & 100 & 85 & 50 & 50 & 50 & 60 & 70 \\
\hline 64 & 50 & 100 & 100 & 90 & 100 & 89 & 50 & 20 & 50 & 70 & 80 \\
\hline 65 & 30 & 90 & 100 & 80 & 100 & 91 & 30 & 90 & 90 & 80 & 80 \\
\hline 66 & 60 & 100 & 100 & 90 & 100 & 92 & 20 & 20 & 20 & 60 & 70 \\
\hline 67 & 50 & 100 & 100 & 90 & 100 & 93 & 20 & 80 & 80 & 70 & 70 \\
\hline 68 & 50 & 100 & 100 & 90 & 100 & 94 & 80 & 90 & 90 & 100 & 100 \\
\hline 69 & 30 & 90 & 100 & 80 & 100 & 95 & 80 & 80 & 80 & 90 & 90 \\
\hline 74 & 70 & 90 & 100 & 100 & 100 & 96 & 80 & 80 & 80 & 90 & 90 \\
\hline 76 & 30 & 80 & 80 & 90 & 90 & 98 & 80 & 80 & 80 & 90 & 100 \\
\hline \multicolumn{7}{|c|}{ Average Precision for these 26 Samples } & 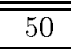 & $\overline{69.2}$ & $\overline{771.1}$ & 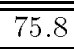 & 87.7 \\
\hline
\end{tabular}

10. C Buckley and G. Salton. Optimization of relevance feedback weights. 18th International ACM SIGIR Conference on Research and Development in Information Retrieval, Seattle, WA, USA, July 1995, pp351-357.

11. Y. Ishikawa, R. Subramanya, C. Faloutsos et al. Mindreader: querying database through multiple examples. Proceedings of the Twenty-Fourth International Conference on Very-Large Databases, New York, Aug. 1998, pp218-227.

12. Y. Rui, T. S. Huang, M. Ortega, and S. Mehrotra. Relevance feedback: a power tool for interactive content-based image retrieval. IEEE Transactions on Circuits and Systems for Video Technology, vol. 8, No. 5, pp644-655, 1998.

13. Y. Rui and T. S. Huang. A novel relevance feedback technique in image retrieval. Proceedings ACM Multimedia '99(Part2),Orlando, FL, USA, 1999, pp67-70.

14. M. E. J. Wood, N. W. Campbell and B. T. Thomas. Iterative refinement by relevance feedback in content-based digital image retrieval. Proceedings ACM Multimedia'98, Bristol, UK, Sept, 1998, pp13-18

15. I. King, Z. Jin and D. Y. M. Chan. Chinese cursive script character image retrieval based on an integrated probability function, submitted to the VISUAL 2000, 4th International Conference on Visual Information Systems, Lyon, France, November $2-4,2000$ 